\title{
Present Status and Future of Opto-Mechatronics*
}

\author{
Kiyoshi ITAO** and Yasuhide NISHIDA**
}

Computer machine systems employing optical technologies have been rapidly increasing in volume in our offices and homes. They are represented by image scanners, laser printers and optical memories. These systems are created by the integration of three basic hardware technologies : mechanics, electronics and optics, and software technologies. In the 1970's, the concept of mechatronics was born by integrating mechanics and electronics. With the recent progress of optical technologies, especially the development of semiconductor lasers the mechatronics concept is being advanced to optomechatronics. Focusing on information systems and measurement systems, the current status of the technology is described and its future trends are predicted.

Key Words : Mechatronics, Optical Engineering, Electronics, Laser, Information Systems, Measurement Systems

\section{Introduction}

Machine systems were quite independent of electronics technology at an earlier stage. With the progress of sensor and processor technologies, however, their relationships have changed, resulting in the development of electromechanical systems which are called mechatronics. With the recent progress of optical technology, especially semiconductor lasers, the mechatronics concept is being advanced to optomechatronics.

Present machine systems are made up of the five elements shown in Fig. 1: sensors, processors, actuators, energy sources and mechanism/structure. However, this has not always been the case. In the mechanics period, when only mechanical technology was used, systems comprised only three elements : mechanism/structure, actuators, and energy sources.

* Received 31st July, 1989

* NTT Applied Electronics Laboratoreis, 3-9-11 Midori-cho, Musashino, Tokyo, 180, Japan
During this period, natural energy sources such as horse power and hydraulic power were used. In the last stage of the mechanics period, electric devices were introduced to actuators and energy sources. These machine systems were called electric machines, but in fact were only aggregations of independent components.

In the next period, processors and sensors utilizing electronics technology were introduced. The five elements were joined systematically, and mechanics and electronics were fused to create mechatronics. This process was accelerated by the advent of micro-

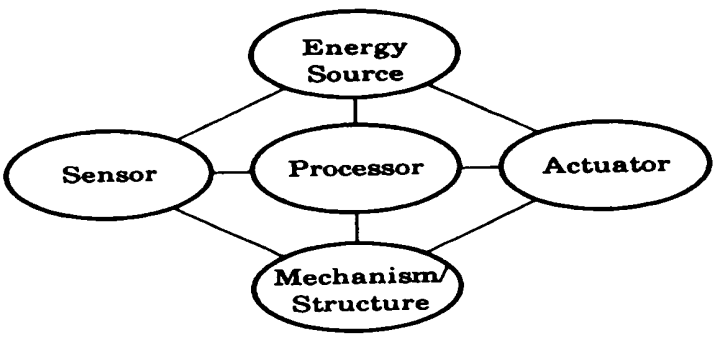

Fig. 1 Elements of machine systems 
processors and the progress of microelectronics. Mechatronics has produced highly valuable results in many machine systems and is now in its heyday.

Now, mechatronics systems which utilize optical devices for sensors and heat energy sources are emerging. They have started to achieve brand new functions and record-breaking performances. With the fusion of optics and mechatronics, the opto-mechatronics period began. (See Fig. 2.)

The new functions are realized, for example, in optical disk drives, bar-code readers, image sensors and laser printers. They utilize optical reading and writing components instead of electrical or mechanical ones. As examples of performance improvements, the realization of sub- $\mu \mathrm{m}$ and sub-sub- $\mu \mathrm{m}$ position control accuracy in superprecision processing machines and optical disk drives is a notable achievement.

The development of lasers played a leading role in realizing these performances and functions. In 1960, MIT succeeded in producing helium-neon emission. In 1970, Bell Laboratories achieved continuous, room-temperature semiconductor laser emission. Since then, various kinds of lasers have been developed and are utilized according to their characteristics. $\mathrm{CO}_{2}{ }^{-}$gas lasers are high powered and mainly used in laser processing. Helium-neon lasers have high coherence and are used for optical measurement. Semiconductor lasers, which are the smallest in size and the lowest in cost, are used in information systems such as optical disk storage and laser printers.

This paper will deal with optics and mechatronics, focusing on information systems and measurement systems technologies.

\section{Optics and Mechatronics in Information Sys- tems}

Information systems have five functions as shown in Fig. 3 : input, transmission, processing, storage and output. Opto-mechatronics and related technologies are widely used for these functions, as shown in Table 1. In this chapter, the present status of the technology

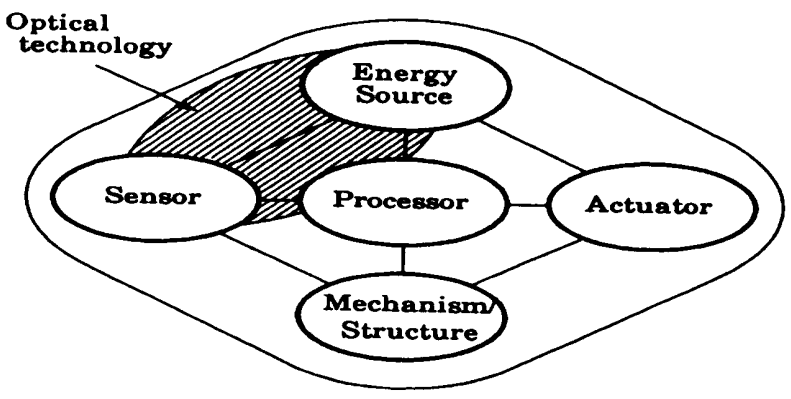

Fig. 2 Optical technology in machine systems in the three functions, input, storage and output, where the technology is most vigorously utilized, will be described.

\section{1 Input device}

Input devices using optical technology such as card readers and tape readers have been in use for many years. More recently, bar-code readers, optical character readers (OCRs) and image scanners have been developed. With these devices, input resolution is greatly improved and the types of input data have diversified to codes, characters and picture images.

The bar-code reader is used in point-of-sale (POS) systems. It optically reads bar-codes, which represent figures and letters, encoded as lines and spaces of different widths. When the bar-codes were standardized in the 1970's, the standardization was based on the wavelength of a $\mathrm{He}-\mathrm{Ne}$ laser, $633 \mathrm{~nm}$, which was then common usage ${ }^{(1)}$. This is why $\mathrm{He}-\mathrm{Ne}$ lasers are used widely even now.

There are two types of bar-code readers : a handheld type and a fixed type. The former, which an operator holds in his/her hand, reads the code by

Table 1 Systems and device technology rerated to optomechatronics

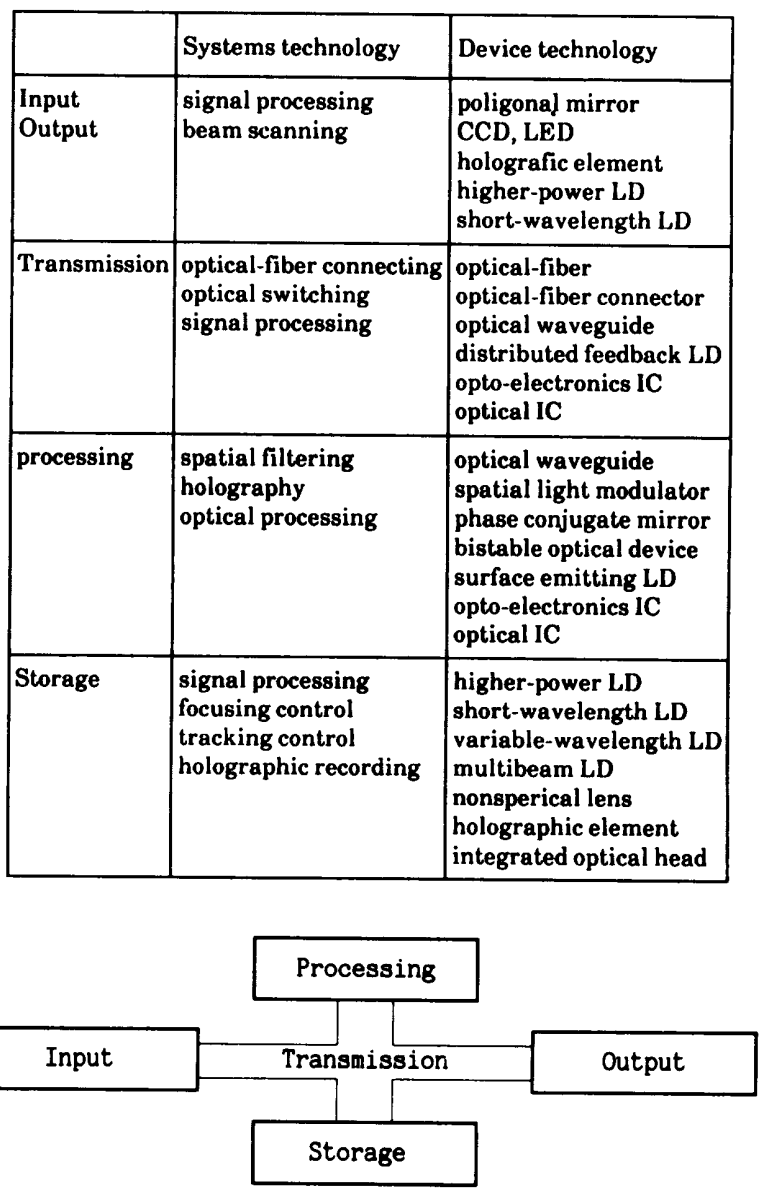

Fig. 3 Function of information systems 
aligning the direction of the light with that of the code. A light-emitting diode (LED) array is generally used as the light source in this type. This hand-held type is used mainly at small stores where space is limited, because it is compact and has no moving parts. On the other hand, the fixed type is positioned on a floor or a stand. Bar-codes are read by moving objects across the reader. Multidirection laser-scanning systems with polygon mirrors or hologram scanners are used to allow flexibility in the direction and passing speed of the bar-code. Hologram scanners utilize a rotating holographic optical element. This element has two functions, deflection and focusing, realizing a compact and low-cost scanning system ${ }^{(2)}$. Recently, a portable fixed-type device of 8 in depth was developed using a fixed holographic element combined with a polygonal mirror ${ }^{(3)}$. A lot of effort is being devoted to making this device still smaller and easier to use. For that purpose, development of shorter-wavelength semiconductor lasers, such as alter. nating $\mathrm{He}-\mathrm{Ne}$ lasers, is essential.

Image scanners are the input devices for twodimensional information. Halogen and tungsten lamps are used as light sources. Image scanners read information in the transverse direction of the sheet using a one-dimensional image sensor such as a CCD or a photo diode array. In the vertical direction, they read information by moving either the paper or the image sensor mechanically. Recently, a compact and solid-state image scanner was developed utilizing light-emitting diode arrays, contact image sensors and self-focusing rod optical fiber lens arrays. The quality of an image scanner is primarily dependent on the resolution of its image sensors. Currently, sensors with $16 \mathrm{dot} / \mathrm{mm}$ resolution are developed and used for high-quality devices. The next target is a compact color scanner with higher resolution.

\subsection{Storage device}

Optical disk storage is superior to other storage forms in recording density, bit cost, media interchangeability, reliability and random accessibility. There are three types of optical disks: read-only, writeonce, and rewritable ones.

The read-only type is mass-produced by a stamping technique and is widely used for video disks and compact disks. The write-once type can be written only once and then used as a read-only type. This type has been used in filing systems to store image data. The rewritable type is an ideal optical disk which allows users to write, read and erase repeatedly. There are two types of recording methods: the magneto-optical method and the phase-change method. Standards for magneto-optical systems using a $130 \mathrm{~mm}$ diameter disk have been agreed in
ISO, and rewritable disks are now commercially available ${ }^{(4)}$.

In optical disk drives, the data on the disk are recorded and detected by a laser beam emitted from a semiconductor laser and focused to a diameter of about $1 \mu \mathrm{m}$ on the surface of the disk. This very small beam spot is positioned and moved to the desired track with an accuracy higher than $1 \mu \mathrm{m}$ in the focusing direction and higher than $0.1 \mu \mathrm{m}$ in the tracking direction. Servo-signals for position control are detected by the same laser beam. That is to say, in an optical disk drive, the laser beam plays three roles simultaneously: a recording power source, a data detecting sensor and a positioning control sensor ${ }^{(5)}$. Optical disk storage is, therefore, the most typical product of opto-mechatronics and could not have been developed without it.

The range of optical disk system applications is now expanding to information processing systems, resulting in digital data storage with rewritable optical disks. In order to expand the application range further, however, data access time and transfer rates must be brought into line with those of magnetic disk storage systems ${ }^{(6)}$. Toward this goal, a shortwavelength semiconductor laser, a multibeam semiconductor laser, a high-performance mechanism/control technique for tracking, and an integrated optical head are being studied and developed.

In addition, some feasibility studies on future recording methods are being carrded out. Application of the photochemical hole-burning ( $\mathrm{PHB}$ ) is expected, by frequency-domain optical storage, to enable high recording density of $100 \sim 1000$ times higher than that of present optical disk storage ${ }^{(7)}$. Writing of nanometer-order pits using the principle of scanning tunneling microscopy (STM) has also been attempted ${ }^{(8)}$. Furthermore, holographic storage is expected to achieve a high transfer rate and functional memory ${ }^{(9)}$.

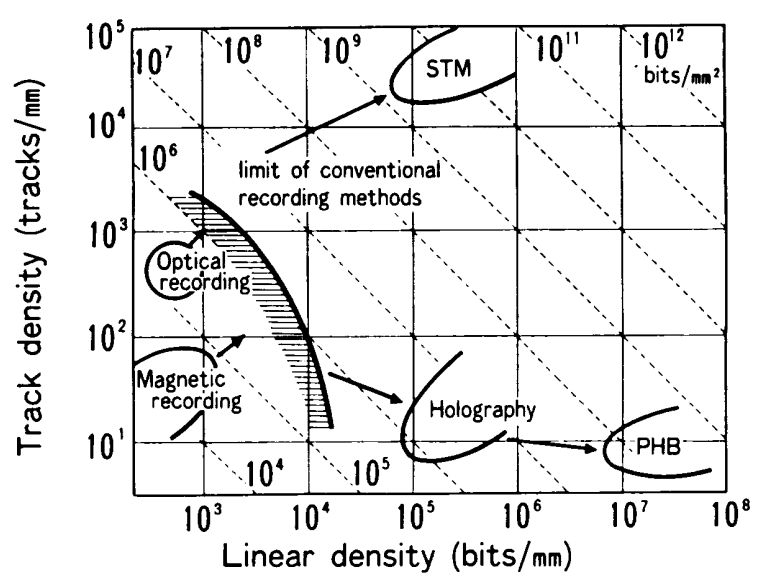

Fig. 4 Recording density at future recording methods 
(Refer to Fig. 4.)

The possibility of the higher storage performance depends on the discovery of new physical phenomena and new materials. To realize new storage systems, however, the development of precise and high-speed control technology proportional to the recording density is also imperative, and opto-mechatronics will play a leading role.

\subsection{Output device}

Laser printers are one of the earliest applications of opto-mechatronics to mechanical systems. These printers utilize laser scanning technology and an electro-photographic recording method. Laser printers have many strong points. Their printing speed is higher than 10000 lines per minute for continuous paper, and more than 260 sheets per minute for cut paper. Moreover, printing quality is higher than 300 dots per inch. Laser printers also offer such benefits as low noise and normal-paper printing.

These printers were developed in the mid-1970's as high-speed nonimpact printers for large computer systems. Figure 5 illustrates a laser subsystem of the printer $^{(10)}$. A gas laser, for example a $\mathrm{He}-\mathrm{Ne}$ or $\mathrm{Ar}$ laser, an acoustic-optical modulator, and a polygonal mirror were used in the system. Later, high-power laser diodes which can be directly modulated, highly sensitive photoconductive materials, and miniaturized optical systems made desktop-type printers possible for office computer systems.

Recently, LED printers and LC printers employing opto-mechatronics technology have also been developed. These types of printers utilize light-emitting diode arrays or liquid-crystal shutter arrays and require no complicated beam scanning mechanism to expose the photoconductor wrapped around the rotatable drum. Hence they offer advantages for miniaturization and cost reduction, and are frequently used as page printers for personal computer systems.

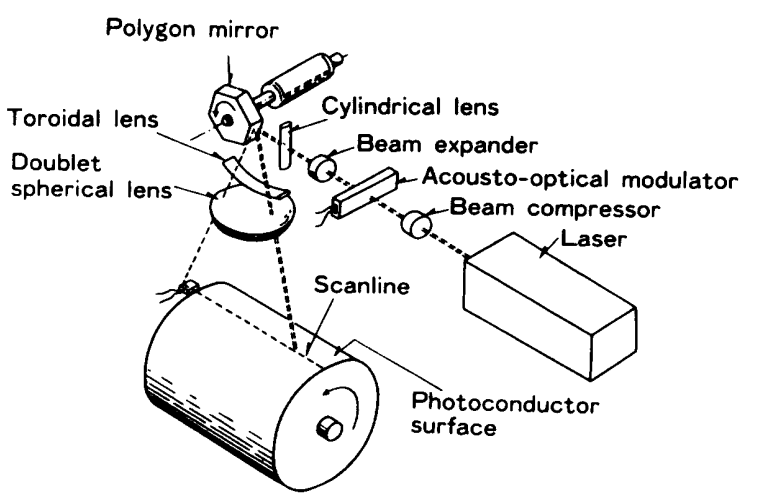

Fig. 5 Laser subsystem of the laser printer ${ }^{(10)}$

\section{Super Accurate Measurement Systems}

Super accurate measurement systems using optical measurement techniques are indispensable for manufacturing, inspection and laboratory equipment. This section presents applications to the development of magnetic disk storages and the processing of LSI's, an area in which optical technology has had the greatest impact on the computer industry.

\subsection{Measurement technique for flying head slider}

In magnetic disk storage systems, a flying head slider is used to support a magnetic transducer above a magnetic medium. Higher recording densities can only be achieved by reducing the spacing between the transducer and medium, and by suppressing the spacing fluctuation ${ }^{(11)}$. Therefore, accurate spacing measurement and evaluation of the dynamic characteristics of slider systems are indispensable for the development of high-performance magnetic disk storage systems.

A highly accurate measurement technique using visible laser interferometry has been developed ${ }^{(12)}$. This measurement system is shown schematically in Fig. 6. A flying head slider is flexibly suspended above the bottom surface of a quartz disk. A helium neon laser beam is focused into a minute $18 \mu \mathrm{m}$ diameter cavity on the slider-medium interface through a beam expander and a focusing lens. The beam reflects off both the slider and disk surfaces, and their interfer-

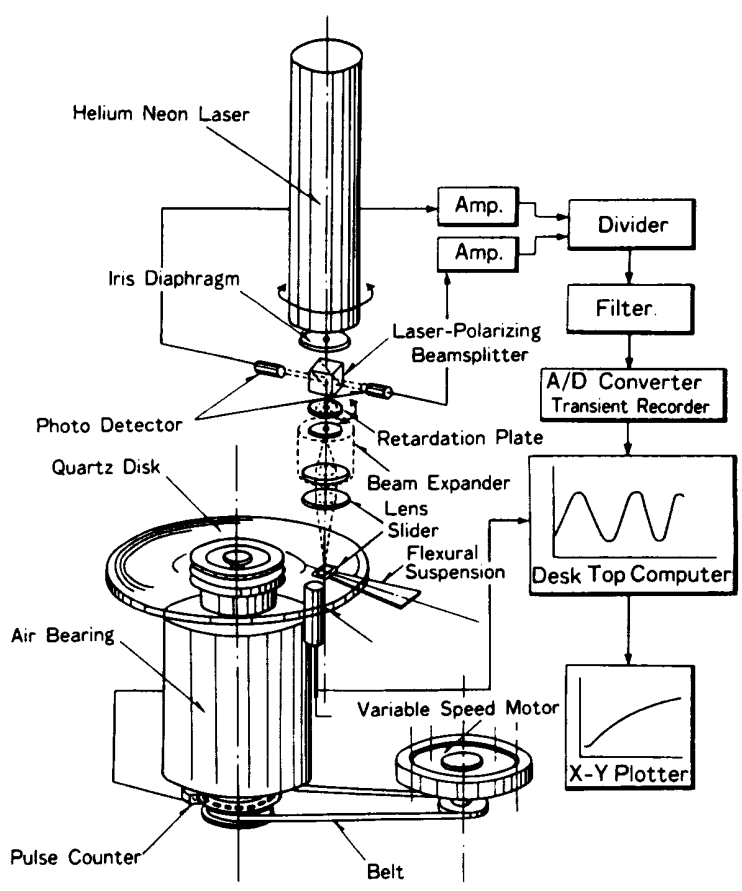

Fig. 6 Measurement system for gas-lubricated slider bearing 
ence produces an intensity variation that depends on the spacing. The intensity variation is converted to an electrical signal by a high-speed PIN silicone photo diode. Laser power drift can be eliminated by virtually monitoring the laser power through a detector and by compensating for the detected interference intensity using an analog divider. This technique achieves a high accuracy down to 1-2 nanometers and a wide frequency range of more than $100 \mathrm{kHz}$.

\section{2 Alignment technique in $\mathrm{X}$-ray exposure sys- tems}

In order to replicate submicron patterns with high through-put, X-ray exposure systems have been actively investigated in many laboratories. One of the most important aspects in an $\mathrm{X}$-ray exposure system using a divergent $\mathrm{X}$-ray is the mask-to-wafer alignment. A precise alignment technique utilizing a single grating and a double-pitch dual grating detection method has been developed ${ }^{(13)}$.

The single grating method has been developed to detect the gap between a mask and a wafer. Figure 7 (a) shows the principle of this method which utilizes a pair of marks consisting of a transparent amplitude grating of pitch $P G$ on the mask and the wafer surface. The gap is measured by detecting the +1 storder diffracted light from the mask grating and the wafer surface. A double-pitch dual grating method has also been developed to detect lateral displace-

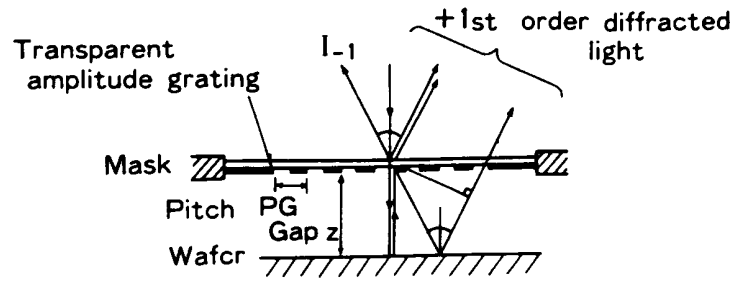

(a) Gap detection principle using a single grating

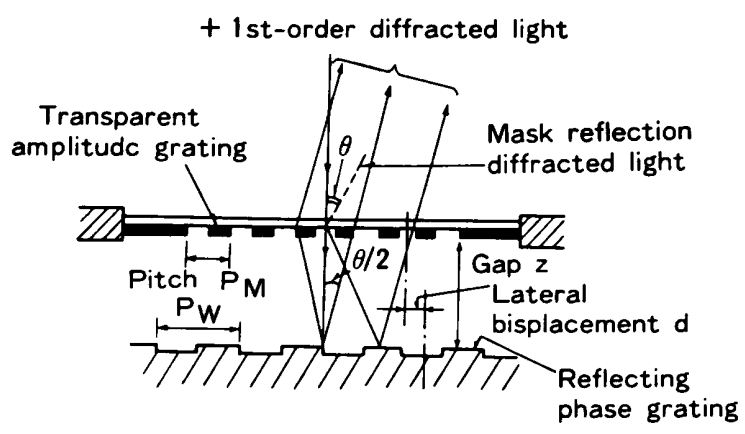

(b) Lateral displacement detection principle using a double-pitch grating

Fig. 7 Precise alignment technique in $\mathrm{X}$-ray exposure system ment. This method utilizes a transparent amplitude grating of pitch $P_{M}$ on the mask and a reflecting phase grating of pitch $P_{W}$ on the wafer, as shown in Fig. 7(b). The pitch $P_{M}$ is twice as fine as $P_{W}$ in order to avoid gap fluctuation influencing on the lateral displacement detection signal. The lateral displacement is measured by detecting the \pm 1 st-order diffracted light from the mask and the wafer gratings.

Detection resolutions of less than $0.01 \mu \mathrm{m}$ were obtained for the gap and lateral displacement. By combining this detection system with the conventional stages, an absolute gap accuracy of $\pm 0.3 \mu \mathrm{m}$ and a lateral alignment servo accuracy of $\pm 0.02 \mu \mathrm{m}$ have been achieved.

\section{Techonology for Advanced Opto-mechatonics}

There are two examples of the technologies for advanced opto-mechatronics in optical disk storage systems and robots.

There are urgent demands for optical disk storage to achieve a higher data transfer rate, a shorter access time, smaller size and lower cost. To meet such requirements, a simpler and smaller optical head must be developed. However, present conventional optical heads have limitations because they are composed of many discrete parts, i. e., optical elements such as lenses, mirrors and beam splitters, and focusing and tracking actuators. Hence a microhead, called an optically switched laser (OSL) head, is proposed. This head exploits the phenomenon of both spontaneous and stimulated emission states of a semiconductor laser ${ }^{(14)}$. In this microhead, a semiconductor laser and a photo diode are attached to the same type of flying slider as in magnetic disk systems. (See Fig. 8.) The spacing fluctuation between the semiconductor laser and the surface of the optical disk is completely suppressed by the air bearing technology, and the focusing servo system is eliminated. This is why this technology can realize a much simpler, smaller, and less expensive optical head. Micromechanics, micro-optics and precise processing technologies are the key technologies for this micro head.

Meanwhile, robots have progressed from a play-

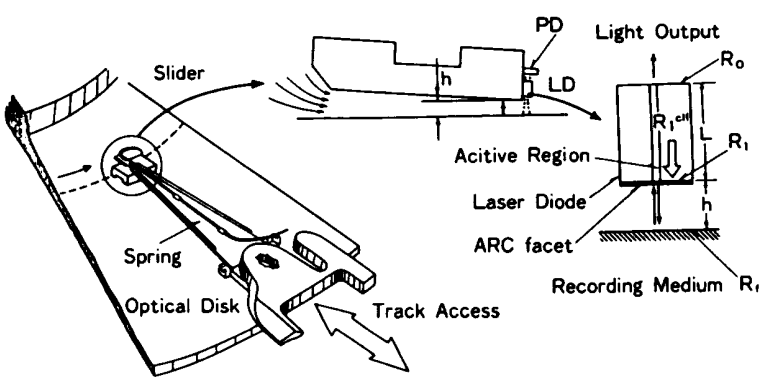

Fig. 8 Optically switched laser (OSL) head 
back type to a feedback type with simple sensors. The next target is an intelligent robot which can understand changes in the environment and adapt its motion sequence accordingly. Visual-information processing is indispensable for the robots ${ }^{(15)}$. The amount of information which has to be dealt with is enormous because it is a group of time variant and three-dimensional image data which has to be processed in real time. Application of such optical technology to information processing will provide the key to these problems. This implies the introduction of optical technology to processors, one of the five elements of machine systems. An eminent characteristic of optical technology, spatial parallelism, will play a leading role in this field. Long-term and vigorous study of materials, devices, algorithms and architectures for optical information processing is anticipated $^{(16)(17)}$.

\section{Conclusions}

Mechanical systems have enhanced additional values by increasing their constituent elements. The technology has evolved from mechanics to mechatronics, moreover, to opto-mechatronics. Though this technology is still young and under development, it is already playing an important role in information systems. The advance of micromechanics and microoptics, which will reduce size and diversify functions of machine systems, will have great impact on the expansion of the application range of opto-mechatronics. Optical processing technology's greatest potential is to enhance the additional value in optomechatronics technology. In the future, opto-mechatronics will become micro-opto-mechatronics.

\section{References}

(1) Sarir, D. and Laurer, G. J., IBM System J., Vol. 14, (1975) p. 16.

(2) Kramer, C. J., SPIE, Vol. 883, (1988) p. 230.

(3) Yamazaki, K., Ichikawa, T., Aritake, H. Yamagishi, F., Ikeda, H. and Inagaki, T. SPIE, Vol. 883, (1988) p. 207.

(4) Itao, K., Yamaji, A., Hara, S. and Izawa, N. Digest of Papers, 8th IEEE Symp. Mass Storage System, (1987) p. 92

( 5 ) Compaan, K. and Kramer, P., J. SMPTE, Vol. 83, (1974) p. 564.

(6) Itao, K. and Hara, S., Jpn. J. Appl. Phys., Vol. 26, Suppl. 26-4, (1974) p. 177.

( 7 ) Schellenberg, F. M., Lenth, W. and Bjorklund, G. C., Appl. Opt., Vol. 25, No. 18, (1986) p. 3207.
(8) Quate, C, F., U.S. Patent, 4,575, 822, (1986).

(9) Dunning, G. J. et al., Opt. Lett., Vol. 12, No. 5 (1987) p. 346.

(10) Fleischer, J. M., Latta, M. R. and Rabedeau, M. E., IBM J. Res. Dev., Vol. 21 (1977) p. 479.

(11) Mitsuya, Y. and Takanami, S., IEEE Trans. Magn., Vol. MAG-23, No. 5, (1987) p. 2674-2679.

(12) Okubo, T. and Kishigami, ASME J. Tribology, Vol. 110, p. 148-155, 1988.

(13) Une, A., Suzuki, M., Okada, I., Saitoh, Y. and Yoshihara, H., SPIE, Vol. 773, (1987) p. 45.

(14) Ukita, H., Katagiri, Y., and Uenishi, Y., Japan. J. Appl. Physics, Vol. 26, Suppl. 26-4, (1987) p. 111.

(15) Brady, M., Computer Vision, Elsevier Science Publishing Company, Netherlands, 1981.

(16) Neff, J. A., Optical Engineering, Vol. 26, No. 1, (1987) p. 2.

(17) Berra, P. B. and Troullinos, N. B., Computer, (1987) p. 59

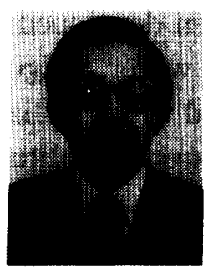

\section{Kiyoshi Itao}

Kiyoshi Itao was born on November 1,1942 . He received the B. S., M. S. and $\mathrm{Ph}$. D. degrees from Tokyo University in 1966, 1968, and 1976, respectively. After joining NTT Electrical Communication Laboratories in 1968, he has carried out research on high-speed precision positioning mechanisms and developments on the impact printers. From 1974 to 1975 he stayed in Massachusetts Institute of Technology as Research Associate. He is presently Executive Manager of Storage Systems Laboratory in the NTT Applied Electronics Laboratories.

Dr. Itao is a member of the Japan Society of Mechanical Engineers, Japan Society of Precision Engineering and the Instite of Electronics, Information and Communication Engineers of Japan.

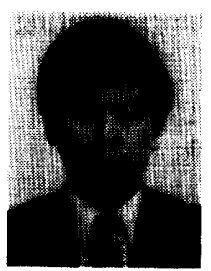

\section{Yasuhide Nishida}

Yasuhide Nishida was born on March 26, 1954. He received the B. S. and M. S. degree from Tohoku University in 1976 and 1978, respectively. $\mathrm{He}$ joined NTT Electrical Communication Laboratories in 1978, where he was engaged in reseach and development on rotating head magnetic tape recording devices. He is presently engaged in research and development on optical storage systems in the NTT Applied Electronics Laboratories.

Mr. Nishida is a member of the Japan Society of Mechanical Engineers and the Japan Society of Applied Physics. 